\title{
ON ROTH'S THEOREM CONCERNING A CUBE AND THREE CUBES OF PRIMES
}

\author{
by XiUmin Ren ${ }^{1}$ AND KAI-MAN Tsang ${ }^{2}$ \\ (Department of Mathematics, The University of Hong Kong, Pokfulam, Hong Kong)
}

\begin{abstract}
In this paper, we prove that with at most $O\left(N^{1271 / 1296+\varepsilon}\right)$ exceptions, all positive integers up to $N$ are the sum of a cube and three cubes of primes. This improves an earlier result $O\left(N^{169 / 170}\right)$ of the first author and the classical result $O\left(N L^{-A}\right)$ of Roth.
\end{abstract}

\section{INTRODUCTION}

It is conjectured that all sufficiently large integers $n$ satisfying some necessary congruence conditions are the sum of four cubes of primes, i.e.

$$
n=p_{1}^{3}+p_{2}^{3}+p_{3}^{3}+p_{4}^{3} .
$$

Such a strong conjecture is out of reach at present; but it is reasonable, in view of the following results of Davenport and Hua respectively. Davenport's theorem in [1] asserts that almost all positive integers are the sum of four positive cubes, while a theorem of Hua $[\mathbf{5}, \mathbf{6}]$ states that almost all positive integers $n$ with $n \not \equiv 0, \pm 2(\bmod 9)$ are the sum of five cubes of primes.

In 1949, Roth [12] proved that almost all positive integers $n$ can be written as

$$
n=m^{3}+p_{2}^{3}+p_{3}^{3}+p_{4}^{3},
$$

where $m$ is a positive integer and $p_{j}$ are primes. To be more precise, we let $E(N)$ denote the number of all the integers $n$ not exceeding $N$ which cannot be written as (1.1). Then Roth's theorem actually states that $E(N) \ll N \log ^{-A} N$, where $A>0$ is arbitrary. This result can be viewed as an approximation to the above conjecture, and the quality of this approximation is indicated in the upper bound of $E(N)$. Roth's theorem has been improved by the first author [11] to $E(N) \ll N^{169 / 170}$. The exponent $169 / 170$ was obtained via an approach in which the possible existence of Siegel zero does not have special influence, and hence the Deuring-Heilbronn phenomenon can be avoided.

In this paper we inject new ideas into the afore-mentioned approach, and make the following further improvement.

Theorem 1.1. For $E(N)$ defined as above, we have

$$
E(N) \ll N^{1271 / 1296+\varepsilon} .
$$

The new ideas used in this paper include the iterative method and the hybrid mean-value estimate for Dirichlet polynomials of Liu [8], which will be displayed in full details at relevant places in the following sections. An outline of the proof of Theorem 1.1 will be given in $\S 2$. At

\footnotetext{
${ }^{1}$ xmren@maths.hku.hk

${ }^{2}$ kmtsang@maths.hku.hk
} 
this stage, we only point out that our Theorem 1.1 does not depend on the Deuring-Heilbronn phenomenon, and the method of this paper can be successfully applied to a number of additive problems.

Notation. As usual, $\varphi(n)$ and $\Lambda(n)$ stand for the function of Euler and von Mangoldt respectively, and $d(n)$ is the divisor function. We use $\chi \bmod q$ and $\chi^{0} \bmod q$ to denote a Dirichlet character and the principal character modulo $q$; and $L(s, \chi)$ is the Dirichlet $L$ function. In our statement, $N$ is a large positive integer, and $L=\log N$. The symbol $r \sim R$ means $R<r \leq 2 R$. The letters $\varepsilon$ and $A$ denote positive constants, which are arbitrarily small and arbitrarily large respectively. We use $c_{j}$ to represent absolute positive constants.

\section{Outline OF The Method}

For large positive integer $N$ and $\theta=25 / 216-\varepsilon$, we set

$$
P=N^{\theta} \quad \text { and } \quad Q=N P^{-1} .
$$

For coprime integers $a, q$ with $1 \leq a \leq q \leq P$, we denote by $\mathfrak{M}(q, a)$ the interval $[a / q-$ $1 / q Q, a / q+1 / q Q]$. These major arcs all lie in $[1 / Q, 1+1 / Q]$ and, since $2 P \leq Q$, they are mutually disjoint. Write $\mathfrak{M}$ for the union of all $\mathfrak{M}(q, a)$ and define the minor arcs $\mathfrak{m}$ as the complement of $\mathfrak{M}$ in $[1 / Q, 1+1 / Q]$.

Let

$$
U=(N / 9)^{1 / 3} \quad \text { and } \quad V=U^{5 / 6}
$$

We define

$$
T(\alpha)=\sum_{m \sim U} e\left(m^{3} \alpha\right)
$$

and, for $W=U$ or $V$,

$$
S(\alpha, W)=\sum_{m \sim W} \Lambda(m) e\left(m^{3} \alpha\right)
$$

Define

$$
r(n)=\sum_{\substack{n=m_{1}^{3}+\ldots+m_{4}^{3} \\ m_{1}, m_{4} \sim U, m_{2}, m_{3} \sim V}} \Lambda\left(m_{1}\right) \Lambda\left(m_{2}\right) \Lambda\left(m_{3}\right) .
$$

Then

$$
r(n)=\int_{1 / Q}^{1+1 / Q} S(\alpha, U) S^{2}(\alpha, V) T(\alpha) e(-n \alpha) d \alpha=\int_{\mathfrak{M}}+\int_{\mathfrak{m}} .
$$

To handle the integral on the major arcs, we need the following.

Lemma 2.1. For all integers $n$ with $N / 2 \leq n \leq N$, we have

$$
\int_{\mathfrak{M}} S(\alpha, U) S^{2}(\alpha, V) T(\alpha) e(-n \alpha) d \alpha=\mathfrak{S}(n) J(n)+O\left(V^{2} U^{-1} L^{-A}\right) .
$$

Here $\mathfrak{S}(n)$ is the singular series which is defined by (4.1) and it satisfies

$$
(\log \log n)^{-c_{1}} \ll \mathfrak{S}(n) \ll \log n
$$


and $J(n)$ is as defined in (4.3) and it satisfies

$$
V^{2} U^{-1} \ll J(n) \ll V^{2} U^{-1}
$$

In the Waring-Goldbach problem, the quality of arithmetical results obtained usually depends on the size of the major arcs. Our major arcs $\mathfrak{M}$ in this paper are much larger than those in [11], and we need new ideas to control their contribution. The first new idea is a hybrid estimate for Dirichlet polynomials; see Lemma 5.1 below. The second is the iterative procedure in treating $I_{1}, \ldots, I_{5}$ in $\S 4$, as illustrated in the proof of Lemma 4.2.

The minor arcs estimate is taken care of by Theorem 3 in [11]. That it is valid for minor arcs as defined above can be checked easily. Here we record it in the following lemma.

Lemma 2.2. Let $\mathfrak{m}$ be as defined above. Then we have

$$
\int_{\mathfrak{m}}|S(\alpha, U)|^{2}|S(\alpha, V)|^{4}|T(\alpha)|^{2} d \alpha \ll U V^{4} P^{-1 / 6+\varepsilon} .
$$

Equipped with Lemmas 2.1 and 2.2, we can now give the proof of Theorem 1.1 immediately.

Proof of Theorem 1.1. We start from (2.3). The contribution of the major arcs is taken care of by Lemma 2.1. To treat the integral on the minor arcs we apply Bessel's inequality and Lemma 2.2 to get

$$
\sum_{N / 2<n \leq N}\left|\int_{\mathfrak{m}}\right|^{2} \ll \int_{\mathfrak{m}}|S(\alpha, U)|^{2}|S(\alpha, V)|^{4}|T(\alpha)|^{2} d \alpha \ll U V^{4} P^{-1 / 6+\varepsilon} .
$$

By a standard argument, we deduce from (2.7) that for all $N / 2<n \leq N$ but at most $O\left(U^{3} P^{-1 / 6+3 \varepsilon}\right)$ exceptions,

$$
\left|\int_{\mathfrak{m}}\right| \ll V^{2} U^{-1} P^{-\varepsilon} .
$$

Therefore by Lemma 2.1, for these un-exceptional $n$, we have

$$
r(n)=\mathfrak{S}(n) J(n)+O\left(V^{2} U^{-1} L^{-A}\right),
$$

and these $n$ can be written as (1.1). Let $F(N)$ be the number of the exceptional $n$ above. Then we have

$$
F(N) \ll U^{3} P^{-1 / 6+3 \varepsilon} \ll N^{1271 / 1296+\varepsilon} .
$$

The assertion of Theorem 1.1 now follows from $E(N)=\sum_{j \geq 0} F\left(N / 2^{j}\right)$.

Now it remains to prove Lemma 2.1, which will be carried out in the following sections.

\section{An EXPLICIT EXPRESSION}

The purpose of this section is to establish in Lemma 3.1 an explicit expression for the left-hand side of (2.4).

For $\chi \bmod q$, we define

$$
C(\chi, a)=\sum_{m=1}^{q} \bar{\chi}(m) e\left(\frac{a m^{3}}{q}\right)
$$


and write $C(q, a)=C\left(\chi^{0}, a\right)$. We also define

$$
S^{*}(q, a)=\sum_{m=1}^{q} e\left(\frac{a m^{3}}{q}\right) .
$$

For $\alpha=a / q+\lambda \in \mathfrak{M}(q, a)$, we have

$$
S(\alpha, W)=\sum_{\substack{h=1 \\(h, q)=1}}^{q} e\left(\frac{a h^{3}}{q}\right) \sum_{\substack{m \sim W \\ m \equiv h(\bmod q)}} \Lambda(m) e\left(\lambda m^{3}\right)+O\left(L^{2}\right) .
$$

By introducing Dirichlet characters to the above sum over $m$, we can rewrite $S(\alpha, W)$ as

$$
\frac{C(q, a)}{\varphi(q)} \sum_{m \sim W} e\left(\lambda m^{3}\right)+\sum_{\chi \bmod q} \frac{C(\chi, a)}{\varphi(q)} \sum_{m \sim W}\left(\Lambda(m) \chi(m)-\delta_{\chi}\right) e\left(\lambda m^{3}\right)+O\left(L^{2}\right) .
$$

Here and throughout, $\delta_{\chi}$ is 1 or 0 according as $\chi$ is principal or not. By Lemma 4.8 in [13] one finds that, for $W=U$ or $V$,

$$
\sum_{m \sim W} e\left(\lambda m^{3}\right)=\int_{W}^{2 W} e\left(\lambda u^{3}\right) d u+O(1) .
$$

Thus, if we denote by $\Phi(\lambda, W)$ the above integral and by $\Psi(\chi, \lambda, W)$ the last sum over $m$ in (3.3), then we have

$$
\begin{aligned}
S(\alpha, W) & =\frac{C(q, a)}{\varphi(q)} \Phi(\lambda, W)+\sum_{\chi \bmod q} \frac{C(\chi, a)}{\varphi(q)} \Psi(\chi, \lambda, W)+O\left(L^{2}\right) \\
& =S_{1}(\lambda, W)+S_{2}(\lambda, W)+O\left(L^{2}\right),
\end{aligned}
$$

say. For $T(\alpha)$ we apply Theorem 4.1 in Vaughan [14], to get

$$
T(\alpha)=\frac{S^{*}(q, a)}{q} \Phi(\lambda, U)+O\left(q^{1 / 2+\varepsilon}\right)=T_{1}(\lambda)+O\left(q^{1 / 2+\varepsilon}\right),
$$

say. So if we write

$$
\Delta(\lambda)=\left\{S_{1}(\lambda, U)+S_{2}(\lambda, U)\right\}\left\{S_{1}(\lambda, V)+S_{2}(\lambda, V)\right\}^{2} T_{1}(\lambda),
$$

then (3.4) and (3.5) together with the trivial bounds $|S(\alpha, W)| \ll W$ and $|T(\alpha)| \ll U$ show that

$$
\left|S(\alpha, U) S^{2}(\alpha, V) T(\alpha)-\Delta(\lambda)\right| \ll U V^{2} q^{1 / 2+\varepsilon}+U^{2} V L^{2} .
$$

Consequently

$$
\begin{aligned}
& \int_{\mathfrak{M}} S(\alpha, U) S^{2}(\alpha, V) T(\alpha) e(-n \alpha) d \alpha \\
& =\sum_{q \leq P} \sum_{\substack{a=1 \\
(a, q)=1}}^{q} e\left(-\frac{a n}{q}\right) \int_{-1 / q Q}^{1 / q Q} \Delta(\lambda) e(-n \lambda) d \lambda+O\left(U V^{2} P^{3 / 2+\varepsilon} Q^{-1}+U^{2} V P Q^{-1} L^{2}\right) .
\end{aligned}
$$

The above $O$-term is $O\left(V^{2} U^{-1} L^{-A}\right)$, on recalling (2.1) and (2.2).

Now we write

$$
\Delta_{0}(\lambda)=S_{1}^{2}(\lambda, V), \quad \Delta_{1}(\lambda)=2 S_{1}(\lambda, V) S_{2}(\lambda, V), \quad \Delta_{2}(\lambda)=S_{2}^{2}(\lambda, V) ;
$$


and for $i=0,1,2$, define

$$
\begin{aligned}
& I_{i}=\sum_{q \leq P} \sum_{\substack{a=1 \\
(a, q)=1}}^{q} e\left(-\frac{a n}{q}\right) \int_{-1 / q Q}^{1 / q Q} S_{1}(\lambda, U) T_{1}(\lambda) \Delta_{i}(\lambda) e(-n \lambda) d \lambda, \\
& I_{3+i}=\sum_{q \leq P} \sum_{\substack{a=1 \\
(a, q)=1}}^{q} e\left(-\frac{a n}{q}\right) \int_{-1 / q Q}^{1 / q Q} S_{2}(\lambda, U) T_{1}(\lambda) \Delta_{i}(\lambda) e(-n \lambda) d \lambda .
\end{aligned}
$$

Then we have proved

Lemma 3.1. For $I_{j}$ defined as above, we have

$$
\int_{\mathfrak{M}} S(\alpha, U) S^{2}(\alpha, V) T(\alpha) e(-n \alpha) d \alpha=\sum_{j=0}^{5} I_{j}+O\left(V^{2} U^{-1} L^{-A}\right) .
$$

In the following sections we prove that $I_{0}$ produces the main term, while the others contribute to the error term.

4. Estimation of $I_{j}$ FOR $j=0,1, \ldots, 5$.

We need some more notations. Let $\chi_{1}, \chi_{2}$ and $\chi_{3}$ be characters $\bmod q, C(\chi, a)$ and $S^{*}(q, a)$ be as defined in (3.1) and (3.2). We define

$$
B\left(n, q, \chi_{1}, \chi_{2}, \chi_{3}\right)=\sum_{\substack{a=1 \\(a, q)=1}}^{q} e\left(-\frac{a n}{q}\right) C\left(\chi_{1}, a\right) C\left(\chi_{2}, a\right) C\left(\chi_{3}, a\right) S^{*}(q, a),
$$

and write

$$
\begin{gathered}
B(n, q)=B\left(n, q, \chi^{0}, \chi^{0}, \chi^{0}\right), \\
A(n, q)=\frac{B(n, q)}{\varphi^{3}(q) q}, \quad \mathfrak{S}(n)=\sum_{q=1}^{\infty} A(n, q) .
\end{gathered}
$$

This $\mathfrak{S}(n)$ is the singular series appearing in Lemma 2.1. By Lemma 18 and 22 in [12] and Lemma 4.4 in $[\mathbf{1 1}]$, we see that the singular series is absolutely convergent and satisfies the first inequality in (2.5). The second inequality in (2.5) can be established by making use of Lemmas 15 and 16 in $[\mathbf{1 2}]$ as follows:

$$
\begin{aligned}
\sum_{q}|A(n, q)| & \ll \prod_{p \mid n}\left(1+c_{2} p^{-1}\right) \prod_{p \nmid n}\left(1+c_{2} p^{-\frac{3}{2}}\right) \\
& \ll \prod_{p \mid n}\left(1+c_{2} p^{-1}\right) \ll\left(\frac{n}{\varphi(n)}\right)^{c_{2}} \ll \log n .
\end{aligned}
$$

Lemma 4.1. Let $I_{0}$ be as defined in (3.6). Then for all $N / 2<n \leq N$, we have

$$
I_{0}=\mathfrak{S}(n) J(n)+O\left(V^{2} U^{-1} L^{-A}\right),
$$

where $\mathfrak{S}(n)$ and $J(n)$ are defined in (4.1) and (4.3). 
Proof. By definition we have

$$
I_{0}=\sum_{q \leq P} \frac{B(n, q)}{\varphi^{3}(q) q} \int_{-1 / q Q}^{1 / q Q} \Phi^{2}(\lambda, U) \Phi^{2}(\lambda, V) e(-n \lambda) d \lambda .
$$

Define

$$
J(n)=\int_{-\infty}^{\infty} \Phi^{2}(\lambda, U) \Phi^{2}(\lambda, V) e(-n \lambda) d \lambda .
$$

Then by Lemma 5.2 in $[\mathbf{1 1}], J(n)$ is well defined and satisfies (2.6). Using the elementary estimate

$$
\Phi(\lambda, W) \leq \min \left(W, \frac{1}{W^{2}|\lambda|}\right)
$$

we get

$$
\int_{|\lambda| \geq 1 / q Q}\left|\Phi^{2}(\lambda, U) \Phi^{2}(\lambda, V)\right| d \lambda \ll V^{2} U^{-4} \int_{1 / q Q}^{\infty} \frac{d \lambda}{\lambda^{2}} \ll V^{2}(U P)^{-1} q,
$$

and therefore,

$$
I_{0}=J(n) \sum_{q \leq P} A(n, q)+O\left\{V^{2}(U P)^{-1} \sum_{q \leq P} q|A(n, q)|\right\} .
$$

By Lemma 18 in [12],

$$
\sum_{q \leq P} A(n, q)=\mathfrak{S}(n)+O\left(P^{-1 / 4+\varepsilon}\right),
$$

so the main term on the right hand-side of (4.5) becomes

$$
J(n) \mathfrak{S}(n)+O\left(V^{2} U^{-1} P^{-1 / 4+\varepsilon}\right) .
$$

To estimate the $O$-term in (4.5), we use the bound (see [12, pp. 277])

$$
|A(n, q)| \ll q^{-3 / 2+\varepsilon}(n, q)^{1 / 2},
$$

to get

$$
\sum_{q \leq P} q|A(n, q)| \ll \sum_{q \leq P} q^{-1 / 2+\varepsilon}(n, q)^{1 / 2} \ll \sum_{d \mid n} d^{\varepsilon} \sum_{q \leq P / d} q^{-1 / 2+\varepsilon} \ll P^{1 / 2+\varepsilon},
$$

and consequently the $O$-term in (4.5) is $O\left(V^{2} U^{-1} P^{-1 / 2+\varepsilon}\right)$. This proves Lemma 4.1.

Lemma 4.2. Let $I_{j}, j=1,2, \ldots, 5$ be as defined in (3.6) and (3.7). Then we have

$$
I_{j} \ll V^{2} U^{-1} L^{-A} \text {. }
$$

To prove Lemma 4.2, we need the following Lemmas 4.3-4.5.

Lemma 4.3. If $\chi_{j}, j=1,2,3$, are primitive characters $\bmod r_{j}$, and $r_{0}=\left[r_{1}, r_{2}, r_{3}\right]$ is the least common multiple of $r_{1}, r_{2}, r_{3}$, then for $\chi^{0} \bmod q$ we have

$$
\sum_{\substack{q \leq P \\ r_{0} \mid q}} \frac{\left|B\left(n, q, \chi_{1} \chi^{0}, \chi_{2} \chi^{0}, \chi_{3} \chi^{0}\right)\right|}{\varphi^{3}(q) q} \ll r_{0}^{-5 / 6+\varepsilon} L
$$


The saving of $r_{0}^{-5 / 6+\varepsilon}$ on the right-hand side will play a key role in our argument, and the quality of the exceptional set will depend on the magnitude of the exponent $5 / 6$. In the next section, we will apply the iterative method of [8] to make use of the full strength of this $5 / 6$, and the reader is referred to the proof of Lemma 4.2.

Proof of Lemma 4.3. This is a slight modification of Lemma 4.5 in [11]. In fact, by (4.7)-(4.10) in [11], we see that

$$
\sum_{\substack{q \leq P \\ r_{0} \mid q}} \frac{\left|B\left(n, q, \chi_{1} \chi^{0}, \chi_{2} \chi^{0}, \chi_{3} \chi^{0}\right)\right|}{\varphi^{3}(q) q} \ll r_{0}^{-5 / 6+\varepsilon} \sum_{i=0}^{2} \sum_{\substack{q \leq P / 3^{i} r_{0} \\\left(q, r_{0}\right)=1}}|A(n, q)| .
$$

From this the desired assertion follows by applying (4.2) to the last sum.

Lemma 4.4. Let $g$ be a positive integer. Then we have

$$
\sum_{r \leq P} \sum_{\chi \bmod r}{ }^{*}[r, g]^{-5 / 6+\varepsilon}\left(\int_{-1 / r Q}^{1 / r Q}|\Psi(\chi, \lambda, U)|^{2} d \lambda\right)^{1 / 2} \ll g^{-5 / 6+\varepsilon} U^{-1 / 2} L^{c_{3}} .
$$

In particular, for $g=1$, the above bound can be improved to $U^{-1 / 2} L^{-A}$. Here $\sum^{*}$ indicates that the summation is over all primitive characters $\bmod r$.

Lemma 4.5. (1) For integer $g \geq 1$, we have

$$
\sum_{r \leq P} \sum_{\chi \bmod r}^{*}[r, g]^{-5 / 6+\varepsilon} \max _{|\lambda| \leq 1 / r Q}|\Psi(\chi, \lambda, V)| \ll g^{-5 / 6+\varepsilon} V L^{17} .
$$

(2) In particular, for $g=1$ the above bound can be improved to $V L^{-A}$.

The proofs of Lemmas 4.4 and 4.5 will be postponed to the next section. With these lemmas ready, we can now give the proof of Lemma 4.2.

Proof of Lemma 4.2. We first consider $I_{5}$. By definition,

$$
\begin{aligned}
I_{5}= & \sum_{q \leq P} \sum_{\chi_{1} \bmod } \sum_{q \chi_{2} \bmod } \sum_{q \chi_{3} \bmod q} \frac{B\left(n, q, \chi_{1}, \chi_{2}, \chi_{3}\right)}{\varphi^{3}(q) q} \\
& \times \int_{-1 / q Q}^{1 / q Q} \Psi\left(\chi_{1}, \lambda, V\right) \Psi\left(\chi_{2}, \lambda, V\right) \Psi\left(\chi_{3}, \lambda, U\right) \Phi(\lambda, U) e(-n \lambda) d \lambda .
\end{aligned}
$$

Reducing the characters into primitive characters and observing that, for primitive character $\chi \bmod r$ with $r \mid q$,

$$
\begin{aligned}
& \left|\Psi\left(\chi \chi^{0}, \lambda, W\right)-\Psi(\chi, \lambda, W)\right| \\
& =\left|\sum_{m \sim W} \Lambda(m)\left(\chi \chi^{0}(m)-\chi(m)\right) e\left(\lambda m^{3}\right)-\left(\delta_{\chi \chi^{0}}-\delta_{\chi}\right) \sum_{m \sim W} e\left(\lambda m^{3}\right)\right| \\
& \leq \sum_{p \mid q, p \nmid r} \sum_{p^{j} \sim W} \log p \ll L,
\end{aligned}
$$


we have

$$
\begin{aligned}
I_{5}= & \sum_{r_{1} \leq P} \cdots \sum_{r_{3} \leq P} \sum_{\chi_{1}}{ }_{\bmod r_{1}}^{*} \cdots \sum_{\chi_{3} \bmod r_{3}} * \sum_{\substack{q \leq P \\
\left[r_{1}, r_{2}, r_{3}\right] \mid q}} \frac{B\left(n, q, \chi_{1} \chi^{0}, \chi_{2} \chi^{0}, \chi_{3} \chi^{0}\right)}{q \varphi^{3}(q)} \\
& \times \int_{-1 / q Q}^{1 / q Q} \Psi\left(\chi_{1} \chi^{0}, \lambda, V\right) \Psi\left(\chi_{2} \chi^{0}, \lambda, V\right) \Psi\left(\chi_{3} \chi^{0}, \lambda, U\right) \Phi(\lambda, U) e(-n \lambda) d \lambda \\
\ll & L \sum_{r_{1} \leq P} \cdots \sum_{r_{3} \leq P} \sum_{\chi_{1} \bmod r_{1}}^{*} \cdots \sum_{\chi_{3} \bmod r_{3}}{ }^{*}\left[r_{1}, r_{2}, r_{3}\right]^{-5 / 6+\varepsilon} I_{5}^{*},
\end{aligned}
$$

by Lemma 4.3. Here

$$
I_{5}^{*}=\int_{-1 /\left[r_{1}, r_{2}, r_{3}\right] Q}^{1 /\left[r_{1}, r_{2}, r_{3}\right] Q} \Psi^{*}\left(\chi_{1}, \lambda, V\right) \Psi^{*}\left(\chi_{2}, \lambda, V\right) \Psi^{*}\left(\chi_{3}, \lambda, U\right)|\Phi(\lambda, U)| d \lambda,
$$

with

$$
\Psi^{*}(\chi, \lambda, W)=|\Psi(\chi, \lambda, W)|+L
$$

By Cauchy's inequality,

$$
\begin{aligned}
I_{5}^{*} \ll & \max _{|\lambda| \leq 1 / r_{1} Q} \Psi^{*}\left(\chi_{1}, \lambda, V\right) \max _{|\lambda| \leq 1 / r_{2} Q} \Psi^{*}\left(\chi_{2}, \lambda, V\right) \\
& \times\left(\int_{-1 / r_{3} Q}^{1 / r_{3} Q}\left(\Psi^{*}\left(\chi_{3}, \lambda, U\right)\right)^{2} d \lambda\right)^{1 / 2}\left(\int_{-1 / Q}^{1 / Q}|\Phi(\lambda, U)|^{2} d \lambda\right)^{1 / 2} .
\end{aligned}
$$

Here an application of (4.4) easily gives

$$
\int_{-1 / Q}^{1 / Q}|\Phi(\lambda, U)|^{2} d \lambda \ll U^{-1} .
$$

On the other hand, by Lemma 4.4 and (4.7),

$$
\begin{aligned}
& \left.\sum_{r_{3} \leq P} \sum_{\chi_{3} \bmod r_{3}}{ }^{*} r_{1}, r_{2}, r_{3}\right]^{-5 / 6+\varepsilon}\left(\int_{-1 / r_{3} Q}^{1 / r_{3} Q}\left(\Psi^{*}\left(\chi_{3}, \lambda, U\right)\right)^{2} d \lambda\right)^{1 / 2} \\
& \ll\left[r_{1}, r_{2}\right]^{-5 / 6+\varepsilon} U^{-1 / 2} L^{c_{3}}+L \sum_{r_{3} \leq P \chi_{3} \bmod r_{3}} \sum^{*}\left[r_{1}, r_{2}, r_{3}\right]^{-5 / 6+\varepsilon}\left(r_{3} Q\right)^{-1 / 2} \\
& \ll\left[r_{1}, r_{2}\right]^{-5 / 6+\varepsilon} U^{-1 / 2} L^{c_{3}} .
\end{aligned}
$$

Collecting these estimates, we get from (4.6) that

$$
\begin{aligned}
I_{5} \ll U^{-1} L^{c_{3}+1} \sum_{r_{1} \leq P} \sum_{\chi_{1} \bmod r_{1}}{ }^{*} \max _{|\lambda| \leq 1 / r_{1} Q} \Psi^{*}\left(\chi_{1}, \lambda, V\right) \\
\quad \times \sum_{r_{2} \leq P \chi_{2}} \sum_{\bmod r_{2}}{ }^{*}\left[r_{1}, r_{2}\right]^{-5 / 6+\varepsilon} \max _{|\lambda| \leq 1 / r_{2} Q} \Psi^{*}\left(\chi_{2}, \lambda, V\right) .
\end{aligned}
$$

By Lemma 4.5 and (4.7), the last double sum is

$$
\ll r_{1}^{-5 / 6+\varepsilon} V L^{17}+L \sum_{r_{2} \leq P} \sum_{\chi_{2} \bmod r_{2}}{ }_{8}^{*}\left[r_{1}, r_{2}\right]^{-5 / 6+\varepsilon} \ll r_{1}^{-5 / 6+\varepsilon} V L^{17} .
$$


Therefore,

$$
\begin{aligned}
I_{5} & \ll U^{-1} V L^{c_{3}+18} \sum_{r_{1} \leq P} \sum_{\chi_{1} \bmod r_{1}}{ }^{*} r_{1}^{-5 / 6+\varepsilon} \max _{|\lambda| \leq 1 / r_{1} Q} \Psi^{*}\left(\chi_{1}, \lambda, V\right) \\
& \ll V^{2} U^{-1} L^{-A}
\end{aligned}
$$

by applying (4.7) and (2) in Lemma 4.5. This proves the lemma for $j=5$.

We remark that, in the iterative argument above, we have used the saving $r_{0}^{-5 / 6+\varepsilon}$ to its full strength, as pointed out right after Lemma 4.3. One sees that the iterative argument is crucial for our improvement, since previously in [11] we used the inequality

$$
r_{0}^{-5 / 6+\varepsilon} \leq r_{1}^{-5 / 18+\varepsilon} r_{2}^{-5 / 18+\varepsilon} r_{3}^{-5 / 18+\varepsilon},
$$

which is responsible for the weaker bound $E(N) \ll N^{169 / 170}$ there. We would also like to add that the key ingredient to fulfil the iterative argument is the hybrid estimate in Lemmas 5.1 and 5.2 below.

To finish the proof of Lemma 4.2, we need to sketch how to estimate $I_{j}$ for $j=1, \ldots, 4$. As an example, we consider $I_{3}$. By definition and by reducing the characters into primitive ones, we get by Lemma 4.3 and (4.7) that

$$
\begin{aligned}
I_{3} & \ll L \sum_{r \leq P} \sum_{\chi \bmod r}{ }^{*} r^{-5 / 6+\varepsilon} \int_{-1 / r Q}^{1 / r Q} \Psi^{*}(\chi, \lambda, U)|\Phi(\lambda, U)|\left|\Phi^{2}(\lambda, V)\right| d \lambda \\
& \ll V^{2} L \sum_{r \leq P} \sum_{\chi \bmod r}{ }^{*} r^{-5 / 6+\varepsilon}\left(\int_{-1 / r Q}^{1 / r Q}\left(\Psi^{*}(\chi, \lambda, U)\right)^{2} d \lambda\right)^{1 / 2}\left(\int_{-1 / Q}^{1 / Q}|\Phi(\lambda, U)|^{2} d \lambda\right)^{1 / 2} \\
& \ll V^{2} U^{-1} L^{-A},
\end{aligned}
$$

by (4.8) and Lemma 4.4 for $g=1$. This finishes the proof of Lemma 4.2.

\section{Proof of Lemmas 4.4 And 4.5 .}

We need to do some preparations to establish Lemmas 4.4 and 4.5. Let $M$ be a large positive integer and let $M_{j}, j=1, \ldots, 10$, be positive integers satisfying

$$
2^{-9} M \leq M_{1} \cdots M_{10} \leq(2 M) \quad \text { and } \quad 2 M_{6}, \ldots, 2 M_{10} \leq(2 M)^{1 / 5} .
$$

For any positive integer $m$, let

$$
a_{j}(m)=\left\{\begin{array}{lll}
\log m, & \text { if } j=1, \\
1, & \text { if } j=2, \ldots, 5 \\
\mu(m), & \text { if } j=6, \ldots, 10 .
\end{array}\right.
$$

We define the following functions of a complex variable $s$ :

$$
f_{j}(s, \chi)=\sum_{m \sim M_{j}} \frac{a_{j}(m) \chi(m)}{m^{s}}, \quad F(s, \chi)=f_{1}(s, \chi) \cdots f_{10}(s, \chi) .
$$

Then we have the following hybrid type estimate for $F(1 / 2+i t, \chi)$, which is Lemma 2.1 in Liu [8]. 
Lemma 5.1. For any $T>0, d \geq 1$ and $1 \leq R \leq M^{2}$, we have

$$
\sum_{\substack{r \sim R \\ d \mid r}} \sum_{\chi \bmod r}^{*} \int_{T}^{2 T}\left|F\left(\frac{1}{2}+i t, \chi\right)\right| d t \ll\left\{\frac{R^{2}}{d} T+\frac{R}{d^{1 / 2}} T^{1 / 2} M^{3 / 10}+M^{1 / 2}\right\} L^{c_{4}} .
$$

We also need zero-density estimates in forms stated in the following lemma.

Lemma 5.2. Let $T \geq 1, Q \geq 1$ and $1 \leq d \leq Q$. Then

$$
\sum_{\substack{q \leq Q \\ d \mid q}} \sum_{\chi \bmod q}^{*} N(\sigma, T, \chi) \ll\left(\frac{Q^{2} T}{d}\right)^{A(\sigma)(1-\sigma)} L^{14}
$$

where for $1 / 2 \leq \sigma<3 / 4, A(\sigma)=3 /(2-\sigma)$; and for $3 / 4 \leq \sigma \leq 1, A(\sigma)=12 / 5+\varepsilon$.

Proof. We observe that under the restriction $d \mid q$, Theorem 12.2 in Montgomery [9] can be restated as follows: For $1 / 2 \leq \sigma \leq 4 / 5$

$$
\sum_{\substack{q \leq Q \\ d \mid q}} \sum_{\chi \bmod q}^{*} N(\sigma, T, \chi) \ll\left(\frac{Q^{2} T}{d}\right)^{3(1-\sigma) /(2-\sigma)} L^{9}
$$

and for $4 / 5 \leq \sigma \leq 1$

$$
\sum_{\substack{q \leq Q \\ d \mid q}} \sum_{\chi \bmod q}^{*} N(\sigma, T, \chi) \ll\left(\frac{Q^{2} T}{d}\right)^{2(1-\sigma) / \sigma} L^{14}
$$

This proves the lemma for $1 / 2 \leq \sigma<3 / 4$ and $5 / 6 \leq \sigma \leq 1$. For $3 / 4 \leq \sigma \leq 5 / 6$, the desired assertion is included in (1.1) of Huxley [7].

Proof of Lemma 4.4. Note that for primitive character $\chi \bmod r, \delta_{\chi}=1$ if $r=1$, and $\delta_{\chi}=0$ otherwise. Hence

$$
\begin{aligned}
& \sum_{1 \leq r \leq P} \sum_{\bmod r}^{*}[r, g]^{-5 / 6+\varepsilon}\left(\int_{-1 / r Q}^{1 / r Q}|\Psi(\chi, \lambda, U)|^{2} d \lambda\right)^{1 / 2} \\
= & g^{-5 / 6+\varepsilon}\left(\int_{-1 / Q}^{1 / Q}\left|\sum_{m \sim U}(\Lambda(m)-1) e\left(\lambda m^{3}\right)\right|^{2} d \lambda\right)^{1 / 2} \\
& +\sum_{1<r \leq P \chi \bmod r} \sum^{*}[r, g]^{-5 / 6+\varepsilon}\left(\int_{-1 / r Q}^{1 / r Q}\left|\sum_{m \sim U} \Lambda(m) \chi(m) e\left(\lambda m^{3}\right)\right|^{2} d \lambda\right)^{1 / 2} \\
= & J_{1}+J_{2},
\end{aligned}
$$


say. By Gallagher's lemma (see [3, Lemma 1]),

$$
\begin{aligned}
\int_{-1 / Q}^{1 / Q}\left|\sum_{m \sim U}(\Lambda(m)-1) e\left(\lambda m^{3}\right)\right|^{2} d \lambda & \ll\left(\frac{1}{Q}\right)^{2} \int_{-\infty}^{\infty}\left|\sum_{\substack{v<m^{3} \leq v+Q \\
m \sim U}}(\Lambda(m)-1)\right|^{2} d v \\
& =\left(\frac{1}{Q}\right)^{2} \int_{U^{3}-Q}^{8 U^{3}}\left|\sum_{\substack{Y_{0}<m \leq X_{0} \\
\text { (n) }}}(\Lambda(m)-1)\right|^{2} d v,
\end{aligned}
$$

where

$$
Y_{0}=\max \left(v^{1 / 3}, U\right), \quad X_{0}=\min \left((v+Q)^{1 / 3}, 2 U\right) .
$$

By the trivial bound

$$
\sum_{Y_{0}<m \leq X_{0}}(\Lambda(m)-1) \ll\left(X_{0}-Y_{0}\right) L \ll U^{-2} Q L,
$$

one derives

$$
J_{1} \ll g^{-5 / 6+\varepsilon} U^{-1 / 2} L .
$$

We now turn to $J_{2}$. Applying Gallagher's lemma as before, the integral in $J_{2}$ is

$$
\ll\left(\frac{1}{r Q}\right)^{2} \int_{U^{3}-r Q}^{8 U^{3}}\left|\sum_{\substack{v<m^{3} \leq v+r Q \\ m \sim U}} \Lambda(m) \chi(m)\right|^{2} d v .
$$

Let

$$
Y=Y(v)=\max \left(v^{1 / 3}, U\right), \quad X=X(v)=\min \left((v+r Q)^{1 / 3}, 2 U\right) .
$$

Then the sum in (5.5) can be rewritten as

$$
\sum_{Y<m \leq X} \Lambda(m) \chi(m)
$$

Now we recall Heath-Brown's identity (see [4, Lemma 1]) for $k=5$, which states that for $m \leq 2 M$,

$$
\Lambda(m)=\sum_{j=1}^{5}\left(\begin{array}{l}
5 \\
j
\end{array}\right)(-1)^{j-1} \sum_{\substack{m_{1} \cdots m_{2 j}=m \\
m_{j+1}, \ldots, m_{2 j} \leq(2 M)^{1 / 5}}}\left(\log m_{1}\right) \mu\left(m_{j+1}\right) \cdots \mu\left(m_{2 j}\right) .
$$

Putting this in (5.7), the sum is written into a linear combination of $O\left(L^{10}\right)$ terms, each of which is of the form

$$
\Sigma(X ; \mathbf{M})=\sum_{\substack{m_{1} \sim M_{1} \\ Y<m_{1} \cdots m_{10} \leq X}} \cdots \sum_{\substack{m_{10} \sim M_{10} \\ \text { nan }}} a_{1}\left(m_{1}\right) \chi\left(m_{1}\right) \cdots a_{10}\left(m_{10}\right) \chi\left(m_{10}\right),
$$

where $a_{i}(m)$ are given by (5.2), and $M_{j}$ are positive integers satisfying (5.1) with $M=U$. Here $\mathbf{M}$ denotes the vector $\left(M_{1}, M_{2}, \ldots, M_{10}\right)$. Therefore we get from (5.4) and (5.5) that

$$
J_{2} \ll \sum_{1<r \leq P} \sum_{\chi \bmod r}^{*}[r, g]^{-5 / 6+\varepsilon} \frac{1}{r Q}\left\{\int_{U^{3}-r Q}^{8 U^{3}}\left(\sum_{\mathbf{M}}|\Sigma(X ; \mathbf{M})|\right)^{2} d v\right\}^{1 / 2} .
$$


Applying Perron's summation formula (see for example [13, Lemma 3.12]) and then shifting the contour to the left, we have

$$
\begin{aligned}
\Sigma(X ; \mathbf{M}) & =\frac{1}{2 \pi i} \int_{1+1 / L-i T}^{1+1 / L+i T} F(s, \chi) \frac{X^{s}-Y^{s}}{s} d s+O\left(\frac{X L^{2}}{T}\right) \\
& =\frac{1}{2 \pi i}\left\{\int_{1+1 / L-i T}^{1 / 2-i T}+\int_{1 / 2-i T}^{1 / 2+i T}+\int_{1 / 2+i T}^{1+1 / L+i T}\right\} F(s, \chi) \frac{X^{s}-Y^{s}}{s} d s+O\left(\frac{X L^{2}}{T}\right),
\end{aligned}
$$

where $F(s, \chi)$ is as defined in (5.3), and $T$ is a parameter satisfying $2 \leq T \leq X$. The integral on the two horizontal segments above is bounded by

$$
\max _{1 / 2 \leq \sigma \leq 1+1 / L}|F(\sigma \pm i T, \chi)| \frac{X^{\sigma}}{T} \ll \frac{U L}{T},
$$

in view of the trivial estimate

$$
|F(\sigma \pm i T, \chi)|=\prod_{j=1}^{10}\left|f_{j}(\sigma \pm i T, \chi)\right| \ll L \prod_{j=1}^{10} M_{j}^{1-\sigma} \ll U^{1-\sigma} L .
$$

Thus,

$$
\Sigma(X ; \mathbf{M})=\frac{1}{2 \pi} \int_{-T}^{T} F\left(\frac{1}{2}+i t, \chi\right) \frac{X^{1 / 2+i t}-Y^{1 / 2+i t}}{1 / 2+i t} d t+O\left(\frac{U L^{2}}{T}\right) .
$$

Moreover, one sees from the estimate

$$
\frac{X^{1 / 2+i t}-Y^{1 / 2+i t}}{1 / 2+i t}=\int_{Y}^{X} u^{-1 / 2+i t} d u \ll X^{1 / 2}-Y^{1 / 2} \ll U^{-5 / 2}(r Q)
$$

and the trivial bound

$$
\frac{X^{1 / 2+i t}-Y^{1 / 2+i t}}{1 / 2+i t} \ll \frac{U^{1 / 2}}{|t|}
$$

that

$$
\frac{X^{1 / 2+i t}-Y^{1 / 2+i t}}{1 / 2+i t} \ll \min \left(\frac{r Q}{U^{5 / 2}}, \frac{U^{1 / 2}}{|t|}\right) \leq U^{1 / 2} \min \left(\frac{r}{P}, \frac{1}{|t|}\right) .
$$

Thus, by taking $T=U$, we obtain

$$
\begin{aligned}
\Sigma(X ; \mathbf{M}) \ll & \frac{r U^{1 / 2}}{P} \int_{|t| \leq P / r}\left|F\left(\frac{1}{2}+i t, \chi\right)\right| d t \\
& +U^{1 / 2} \int_{\substack{P / r<|t| \leq U \\
12}}\left|F\left(\frac{1}{2}+i t, \chi\right)\right| \frac{d t}{|t|}+O\left(L^{2}\right) .
\end{aligned}
$$


Note that the right hand side is independent of $v$. Thus by inserting this in (5.8), we obtain

$$
\begin{aligned}
J_{2} \ll & U^{2}(Q P)^{-1} \sum_{1<r \leq P} \sum_{\chi \bmod r}{ }^{*}[r, g]^{-5 / 6+\varepsilon} \sum_{\mathbf{M}} \int_{|t| \leq P / r}\left|F\left(\frac{1}{2}+i t, \chi\right)\right| d t \\
& +U^{2} Q^{-1} \sum_{1<r \leq P} \sum_{\chi \bmod r}{ }^{*} r^{-1}[r, g]^{-5 / 6+\varepsilon} \sum_{\mathbf{M}} \int_{P / r<|t| \leq U}\left|F\left(\frac{1}{2}+i t, \chi\right)\right| \frac{d t}{|t|} \\
& +U^{3 / 2} Q^{-1} L^{12} \sum_{1<r \leq P} \sum{ }^{*}{ }^{*} r^{-1}[r, g]^{-5 / 6+\varepsilon} r \\
= & J_{21}+J_{22}+J_{23},
\end{aligned}
$$

say. Clearly

$$
J_{23} \ll U^{3 / 2} Q^{-1} L^{12} g^{-5 / 6+\varepsilon} \sum_{1<r \leq P} \sum_{\bmod r}{ }^{*} r^{-1} \ll g^{-5 / 6+\varepsilon} U^{-3 / 2} P^{2} L^{12} \ll g^{-5 / 6+\varepsilon} U^{-1 / 2} .
$$

To estimate $J_{21}$, one notes that $[r, g]=r g(r, g)^{-1}$. Thus

$$
\begin{aligned}
J_{21} \ll & U^{-1} g^{-5 / 6+\varepsilon} \sum_{\mathbf{M}} \sum_{d \mid g} d^{5 / 6-\varepsilon} \sum_{\substack{1<r \leq P \\
d \mid r}} \sum_{\chi \bmod r}{ }^{*} r^{-5 / 6+\varepsilon} \int_{|t| \leq P / r}\left|F\left(\frac{1}{2}+i t, \chi\right)\right| d t \\
\ll & g^{-5 / 6+\varepsilon} U^{-1} L \sum_{\mathbf{M}} \sum_{d \mid g} d^{5 / 6-\varepsilon} \max _{d \leq R \leq P} R^{-5 / 6+\varepsilon} \\
& \times \sum_{\substack{r \sim R \\
d \mid r}} \sum_{\chi \bmod r}^{*} \int_{|t| \leq P / R}\left|F\left(\frac{1}{2}+i t, \chi\right)\right| d t .
\end{aligned}
$$

By Lemma 5.1, the last double sum is

$$
\begin{aligned}
& \ll \max _{1 \leq T \leq P / R} \sum_{\substack{r \sim R \\
d \mid r}} \sum_{\chi \bmod r}^{*} \int_{T}^{2 T}\left|F\left(\frac{1}{2}+i t, \chi\right)\right| d t \\
& \ll L^{c_{4}+1}\left\{P R / d+(P R / d)^{1 / 2} U^{3 / 10}+U^{1 / 2}\right\},
\end{aligned}
$$

and hence

$$
J_{21} \ll g^{-5 / 6+\varepsilon} U^{-1} L^{c_{4}+12}\left(P^{7 / 6+\varepsilon}+P^{1 / 2} U^{3 / 10}+U^{1 / 2}\right) \ll g^{-5 / 6+\varepsilon} U^{-1 / 2} L^{c_{4}+12},
$$

on noting that $P \ll U^{2 / 5}$.

Now it remains to estimate $J_{22}$. We have

$$
\begin{aligned}
J_{22} \ll & g^{-5 / 6+\varepsilon} U^{-1} P L \sum_{\mathbf{M}} \sum_{d \mid g} d^{5 / 6-\varepsilon} \max _{d \leq R \leq P} R^{-11 / 6+\varepsilon} \\
& \times \sum_{\substack{r \sim R \\
d \mid r}} \sum_{\chi \bmod r}^{*} \int_{P / 2 R \leq|t| \leq U}\left|F\left(\frac{1}{2}+i t, \chi\right)\right| \frac{d t}{|t|} .
\end{aligned}
$$


The last double sum can be estimated via Lemma 5.1 again, which gives

$$
\begin{aligned}
& \ll \max _{P / 2 R<T \leq U} \frac{1}{T} \sum_{\substack{r \sim R \\
d \mid r}} \sum_{\chi \bmod r} * \int_{T}^{2 T}\left|F\left(\frac{1}{2}+i t, \chi\right)\right| d t \\
& \ll L^{\left(c_{4}+1\right)}\left\{R^{2} d^{-1}+R^{3 / 2}(d P)^{-1 / 2} U^{3 / 10}+R P^{-1} U^{1 / 2}\right\} .
\end{aligned}
$$

Inserting this in (5.9), we get

$$
\begin{aligned}
J_{22} & \ll g^{-5 / 6+\varepsilon} U^{-1} P L^{\left(c_{4}+12\right)}\left(P^{1 / 6+\varepsilon}+P^{-1 / 2} U^{3 / 10}+P^{-1} U^{1 / 2}\right) \\
& \ll g^{-5 / 6+\varepsilon} U^{-1 / 2} L^{c_{4}+12} .
\end{aligned}
$$

The first assertion of Lemma 4.4 now follows by letting $c_{3}=c_{4}+12$.

Now we consider the special case of $g=1$. We write

$$
\sum_{1 \leq r \leq P} \sum_{\chi \bmod r}{ }^{*} r^{-5 / 6+\varepsilon}\left(\int_{-1 / r Q}^{1 / r Q}|\Psi(\chi, \lambda, U)|^{2} d \lambda\right)^{1 / 2}=H_{1}+H_{2}
$$

where $H_{1}, H_{2}$ denote contributions from those with $r \leq L^{B}$ and $L^{B}<r \leq P$, respectively with $B=10 A$. To estimate $H_{2}$, we follow the argument in estimating $J_{2}$ but let $g=1$ and add the restriction $r>L^{B}$. We will get

$$
H_{2} \ll U^{-1 / 2} L^{-A} \text {. }
$$

To estimate $H_{1}$, we apply Gallager's lemma as before to get

$$
H_{1} \ll \sum_{r \leq L^{B}} \sum_{\chi \bmod r}{ }^{*} r^{-5 / 6+\varepsilon} \frac{1}{r Q}\left(\int_{U^{3}-r Q}^{8 U^{3}}\left|\sum_{Y<m \leq X}\left(\Lambda(m) \chi(m)-\delta_{\chi}\right)\right|^{2} d v\right)^{1 / 2}
$$

where $X, Y$ are as defined by (5.6).

Now we apply the explicit formula (see [2, $117,(9)-(10) ; \S 19,(4)-(9)]$ )

$$
\sum_{m \leq x} \Lambda(m) \chi(m)=\delta_{\chi} x-\sum_{|\gamma| \leq T} \frac{x^{\rho}}{\rho}+O\left(\frac{x(\log q x T)^{2}}{T}\right),
$$

where $2<T \leq x$ is a parameter and $\rho=\sigma+i \gamma$ is a typical nontrivial zero of the Dirichlet $L$-function $L(s, \chi)$. Let $T=P L^{2 B}$. Then

$$
\begin{aligned}
\sum_{Y<m \leq X}\left(\Lambda(m) \chi(m)-\delta_{\chi}\right) & \ll \sum_{|\gamma| \leq P L^{2 B}} \frac{\left|X^{\rho}-Y^{\rho}\right|}{|\rho|}+O\left(U P^{-1} L^{-2 B+2}\right) \\
& \ll\left(U^{-2} r Q\right) \sum_{|\gamma| \leq P L^{2 B}} U^{\sigma-1}+O\left(U P^{-1} L^{-2 B+2}\right) .
\end{aligned}
$$

Hence

$$
H_{1} \ll U^{-1 / 2} \sum_{r \leq L^{B}} \sum_{\chi \bmod r}^{*} \sum_{\substack{|\gamma| \leq P L^{2 B} \\ 14}} U^{\sigma-1}+U^{-1 / 2} L^{-A}
$$


By Satz VIII.6.2 of Prachar [10] and Siegel's theorem (see $[\mathbf{2}, \S 21]$ ), there exists a positive constant $c_{5}$ such that for $r \leq L^{B}, \prod_{\chi \bmod r} L(s, \chi)$ is zero-free in the region

$$
\sigma \geq 1-c_{5} / \max \left\{\log r, \log ^{4 / 5} x\right\}, \quad|t| \leq x .
$$

Let $\eta(N)=c_{5} \log ^{-4 / 5} N$. Then by integrating by parts and Lemma 5.2 with $d=1$, we have

$$
H_{1} \ll U^{-1 / 2} L^{15} \max _{1 / 2 \leq \sigma \leq 1-\eta(N)}\left(L^{4 B} P\right)^{(12 / 5+\varepsilon)(1-\sigma)} U^{\sigma-1}+U^{-1 / 2} L^{-A} \ll U^{-1 / 2} L^{-A},
$$

since $P \ll U^{5 / 12-\varepsilon}$.

Proof of Lemma 4.5. (1) By integrating by parts and noticing $|\lambda| V^{3}<1$, we have

$$
\begin{aligned}
\Psi(\chi, \lambda, V) & =\int_{V}^{2 V} e\left(\lambda u^{3}\right) d \sum_{V<m \leq u}\left(\Lambda(m) \chi(m)-\delta_{\chi}\right) \\
& \ll \max _{V \leq u \leq 2 V}\left|\sum_{V<m \leq u}\left(\Lambda(m) \chi(m)-\delta_{\chi}\right)\right| .
\end{aligned}
$$

By applying (5.10) with $T=x / 2=V$ to (5.11), we get

$$
\begin{aligned}
& \sum_{r \leq P} \sum_{\chi \bmod r}{ }^{*}[r, g]^{-5 / 6+\varepsilon} \max _{|\lambda| \leq 1 / r Q}|\Psi(\chi, \lambda, V)| \\
& \ll \sum_{r \leq P} \sum_{\chi \bmod r}^{*}[r, g]^{-5 / 6+\varepsilon} \sum_{|\gamma| \leq V}(1+|\gamma|)^{-1} V^{\beta}+g^{-5 / 6+\varepsilon} P^{2} L^{2} \\
& \ll g^{-5 / 6+\varepsilon} V L^{2} \sum_{\substack{d \leq P \\
d \mid g}} d^{5 / 6-\varepsilon} \max _{d \leq R \leq P} R^{-5 / 6+\varepsilon} \\
& \quad \times \max _{0<T<V}(1+T)^{-1} \sum_{\substack{r \sim R \\
d \mid r}} \sum_{\bmod r}^{*} \sum_{|\gamma| \sim T} V^{\beta-1}+g^{-5 / 6+\varepsilon} V L^{-A} \\
& :=K+g^{-5 / 6+\varepsilon} V L^{-A},
\end{aligned}
$$

say. By making use of Lemma 5.2, we have

$$
\begin{aligned}
& \sum_{\substack{r \sim R \\
d \mid r}} \sum_{\chi \bmod r}{ }^{*} \sum_{|\gamma| \sim T} V^{\beta-1} \\
& \ll L^{14}\left(R^{2} T / d\right)^{A(1 / 2) / 2} V^{-1 / 2}+L^{15} \int_{1 / 2}^{1}\left(R^{2} T / d\right)^{A(\sigma)(1-\sigma)} V^{\sigma-1} d \sigma,
\end{aligned}
$$

where for $1 / 2 \leq \sigma<3 / 4, A(\sigma)=3 /(2-\sigma)$; while for $3 / 4 \leq \sigma \leq 1, A(\sigma)=12 / 5+\varepsilon$. Since $A(\sigma)(1-\sigma) \leq 1$ for $1 / 2 \leq \sigma \leq 1$, we see that the total power of $T$ in $K$ is negative. Thus

$$
K \ll g^{-5 / 6+\varepsilon} V L^{17} \max _{1 / 2 \leq \sigma \leq 1} \sum_{\substack{d \leq P \\ d \mid g}} d^{5 / 6-\varepsilon} \max _{d \leq R \leq P} R^{-5 / 6+\varepsilon}\left(R^{2} / d\right)^{A(\sigma)(1-\sigma)} V^{\sigma-1}
$$


Let $\sigma_{0}=(8+12 \varepsilon) /(13+6 \varepsilon)$, which is the solution of $A(\sigma)(1-\sigma)=5 / 6-\varepsilon$. Then

$$
\begin{gathered}
K \ll g^{-5 / 6+2 \varepsilon} V L^{17} \max _{1 / 2 \leq \sigma \leq \sigma_{0}} P^{2 A(\sigma)(1-\sigma)-5 / 6+\varepsilon} V^{\sigma-1} \\
+g^{-5 / 6+2 \varepsilon} V L^{17} \max _{\sigma_{0}<\sigma \leq 1} P^{A(\sigma)(1-\sigma)} V^{\sigma-1} .
\end{gathered}
$$

The first maximum is $O\left(L^{-A}\right)$ if $P \leq V^{1 / h-\varepsilon}$ where $h=\max _{1 / 2 \leq \sigma \leq \sigma_{0}}\{2 A(\alpha)-5 / 6(1-\sigma)\}=$ $7 / 3$. Similarly the second maximum is $O(1)$ if $P \leq V^{1 / h^{\prime}}$ where $h^{\prime}=\max _{\sigma_{0} \leq \sigma \leq 1} A(\alpha)=$ $12 / 5+\varepsilon$. This proves $(1)$.

(2) We write

$$
\sum_{r \leq P} \sum_{\chi \bmod r}{ }^{*} r^{-5 / 6+\varepsilon} \max _{|\lambda| \leq 1 / r Q}|\Psi(\chi, \lambda, V)|=Z_{1}+Z_{2}
$$

where $Z_{1}$ and $Z_{2}$ denote contributions from those with $r \leq L^{B}$ and $L^{B}<r \leq P$, respectively.

By similar arguments as those leading to (5.12) but with $g=1, d=1$ and the restriction that $R \geq L^{B}$, we get

$$
\begin{aligned}
Z_{2} & \ll V L^{17} \max _{1 / 2 \leq \sigma \leq 1} \max _{L^{B} \leq R \leq P} R^{2 A(\sigma)(1-\sigma)-5 / 6+\varepsilon} V^{\sigma-1} \\
& \ll V L^{17} \max _{1 / 2 \leq \sigma \leq \sigma_{0}} P^{2 A(\sigma)(1-\sigma)-5 / 6+\varepsilon} V^{\sigma-1} \\
& \quad+V L^{17} \max _{\sigma_{0}<\sigma \leq 17 / 18} P^{A(\sigma)(1-\sigma)} V^{\sigma-1}+V L^{-B / 2+17} \\
& \quad \ll L^{-A},
\end{aligned}
$$

since $P \ll V^{5 / 12-\varepsilon}$. This finishes the estimate for $Z_{2}$.

Now we turn to $Z_{1}$. By (5.11), we have

$$
Z_{1} \ll \sum_{r \leq L^{B}} \sum_{\chi \bmod r}^{*} \max _{V \leq u \leq 2 V}\left|\sum_{V<m \leq u}\left(\Lambda(m) \chi(m)-\delta_{\chi}\right)\right| .
$$

Now the desired estimate follows by applying Siegel-Walfisz theorem in the form of the bound: For a primitive character $\chi \bmod r, r \leq \log ^{B} X$

$$
\sum_{m \leq X}\left(\Lambda(m) \chi(m)-\delta_{\chi}\right) \ll X \exp (-c(B) \sqrt{\log X})
$$

This proves the lemma.

Acknowledgement. The authors are indebted to the referee for valuable suggestions that simplify parts of the argument and bring improvements to the paper. The original version of the paper has the result that $E(N) \ll N^{53 / 54+\epsilon}$. The improvement obtained in the present Theorem 1.1 is due to an idea of the referee.

The first author is supported by a Post-Doctoral Fellowship of The University of Hong Kong. 


\section{REFERENCES}

[1] H. Davenport, On Waring's problem for cubes, Acta Math. 71 (1939), 123-143.

[2] H. Davenport, Multiplicative Number Theory, 2nd edition, Springer Verlag, Berlin 1980.

[3] P.X. Gallagher, A large sieve density estimate near $\sigma=1$, Invent. Math. 11 (1970), 329-339.

[4] D.R. Heath-Brown, Prime numbers in short intervals and a generalized Vaughan's identity, Can. J. Math. 34 (1982), 1365-1377.

[5] L.K. Hua, Some results in additive prime number theory, Quart. J. Math. 9 (1938), 68-80.

[6] L.K. Hua, Additive theory of prime numbers, Science press, Beijing 1957; English version, American Mathematical Society, Providence 1965.

[7] M.N. Huxley, Large values of Dirichlet polynomials, III, Acta Arith. 26 (1975), 435-444.

[8] J.Y. Liu, On Lagrange's theorem with prime variables, to appear in Quart. J. Math.

[9] H.L. Montgomery, Topics in Multiplicative number theory, LNM 227, Springer-Verlag, Berlin. Heidelberg. New York 1971.

[10] K. Prachar, Primzahlverteilung, Springer Verlag, Berlin 1957.

[11] X. Ren, The exceptional set in Roth's theorem concerning a cube and three cubes of primes, Quart. J. Math. 52 (2001), 107-126.

[12] K.F. Roth, On Waring's problem for cubes, Proc. London Math. Soc. (2) 53 (1951), 268-279.

[13] E.C. Titchmarsh (Revised by D.R. Heath-Brown), The theory of the Riemann zeta-function, 2nd edition, Oxford University Press, Oxford 1986.

[14] R.C. Vaughan, The Hardy-Littlewood method, 2nd edition, Cambridge University Press, Cambridge 1997. 